CASE REPORT

\title{
Delayed presentation and diagnosis of metastatic hepatocellular carcinoma in pregnancy
}

C N Mnyani, ${ }^{1}$ BA, MB ChB, FCOG (SA); J C Hull, ${ }^{1}$ MB BCh, MRCOG, FCOG (SA), DTM\&H; M B Mbakaza, ${ }^{2}$ MB ChB, FC Rad Diag (SA); A O A Krim, ${ }^{2}$ MB ChB, FC Rad Diag (SA); E Nicolaou, ${ }^{1,3} \mathrm{MD}$, FCOG (SA), Dip Fet Med

\author{
${ }^{1}$ Department of Obstetrics and Gynaecology, Faculty of Health Sciences, Chris Hani Baragwanath Academic Hospital, \\ University of the Witwatersrand, Johannesburg, South Africa \\ ${ }^{2}$ Department of Radiology, Faculty of Health Sciences, Chris Hani Baragwanath Academic Hospital, University of the Witwatersrand, \\ Johannesburg, South Africa \\ ${ }^{3}$ Maternal Fetal Medicine Centre, Morningside MediClinic, Johannesburg, South Africa
}

Correspondingauthor: C N Mnyani (coceka.mnyani@wits.ac.za)

Hepatocellular carcinoma (HCC) is rare in women of reproductive age. If diagnosed, the underlying cirrhosis is associated with infertility in the majority of cases. There is limited literature on HCC in pregnancy, even more so for cases of metastatic disease. We present a case of delayed presentation and diagnosis of metastatic HCC in pregnancy.

A 30-year-old pregnant woman presented at 23 weeks' gestation and was diagnosed as HIV-infected, with anaemia. She was initiated on an efavirenz-based fixed-dose combination and oral haematinics. She subsequently presented at 32 weeks' gestation with dyspnoea, and was diagnosed with pre-eclampsia. She was also found to have hepatosplenomegaly and ascites. She went into spontaneous preterm labour at 32 weeks and 4 days. A diagnosis of metastatic HCC was made postpartum, based on the radiological findings and biochemistry. We discuss the challenges of diagnosing metastatic HCC in pregnancy.

S Afr Med J 2015;105(10):877. DOI:10.7196/SAMJnew.8781

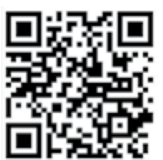

Hepatocellular carcinoma (HCC) is rarely diagnosed in women of reproductive age. The major risk factors are hepatitis B and C infections, alcoholic liver disease, and non-alcoholic fatty liver disease associated with obesity and type 2 diabetes. ${ }^{[1]}$ Most risk factors are associated with progression to cirrhosis, which is present in $80-90 \%$ of cases of HCC. ${ }^{[1]}$ Cirrhosis is associated with infertility; hence, HCC is rarely diagnosed in pregnancy. ${ }^{[1]}$ The literature suggests that pregnancy has an adverse effect on HCC, and that high levels of oestrogen associated with pregnancy may accelerate evolution. ${ }^{[2,3]}$ Therefore, the discussions often focus around termination of pregnancy if the diagnosis is made during pregnancy. ${ }^{[2,3]}$ The finding of a hepatic lesion in pregnancy is often incidental, and the diagnosis of early-stage HCC may be delayed as the symptoms are often nonspecific. ${ }^{[4]}$ We report a case of delayed presentation and diagnosis of metastatic HCC in pregnancy.

\section{Case presentation}

A 30-year-old primiparous patient, with an uneventful previous pregnancy delivered by caesarean section, presented for the first time for antenatal care at 23 weeks' gestation at a primary healthcare facility. She was found to be anaemic, with a haemoglobin of $8.0 \mathrm{~g} / \mathrm{dL}$, and HIV-infected with a CD4 count of 183 cells $/ \mu \mathrm{L}$. She weighed $49.8 \mathrm{~kg}$, with a body mass index of $22.2 \mathrm{~kg} / \mathrm{m}^{2}$. No other abnormal clinical findings were recorded at the first antenatal visit. She was initiated on antiretroviral therapy with an efavirenz-based fixed-dose combination, and also started on oral haematinics. She was referred to the local hospital for assessment, as she had had a previous caesarean delivery.

At the referral hospital, a full blood count was done to investigate the anaemia. The results were not checked until 9 weeks later, when she was found to have a thrombocytopenia of $86 \times 10^{9} / \mathrm{L}$ and a haemoglobin of $11.8 \mathrm{~g} / \mathrm{dL}$. In the interim, the patient had 3 follow-up visits at the local clinic and the referral hospital, and no abnormal clinical findings were detected.
She was again referred to the hospital for pre-eclampsia at 32 weeks' gestation, with a blood pressure of $144 / 105 \mathrm{mmHg}$ and $1+$ proteinuria. There was also a history of shortness of breath. On clinical assessment, she was found to have pitting oedema extending to the level of the thighs bilaterally, and was noted to have ascites and an enlarged liver and spleen. A chest radiograph showed bilateral multiple nodules, and the patient was started on empiric tuberculosis treatment. She went into spontaneous preterm labour at 32 weeks and 4 days and delivered a live male baby, with a birth weight of $1525 \mathrm{~g}$.

Postpartum the patient developed pyrexia of $38.8^{\circ} \mathrm{C}$, a cough and right upper-quadrant pain. An abdominal ultrasound was done,

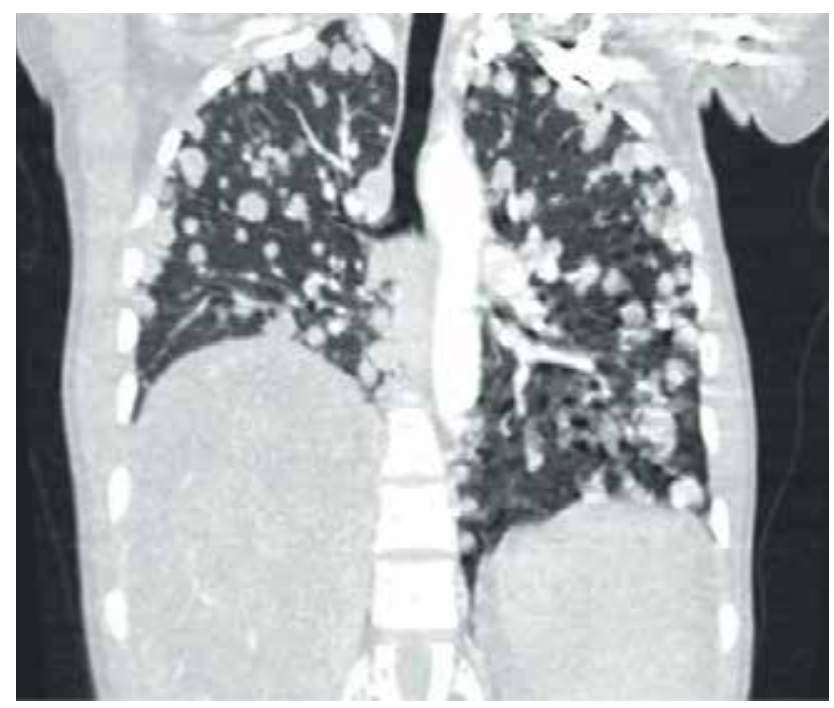

Fig. 1. A CT scan of the chest (coronal view), showing bilateral cannon ball lesions (white lesions) in the lung fields (CT = computed tomography). 
which showed a large lobulated hepatic mass with central lakes and a whorled appearance. There was also evidence of splenomegaly and ascites. A subsequent contrast-enhanced computed tomography (CT) scan of the chest and abdomen showed multiple cannon ball lesions (Fig. 1) in both lungs and an elevated right hemidiaphragm. There was a vascular mass with central areas of breakdown in the right lobe of the liver, measuring $12.3 \mathrm{~cm} \times 19.0 \mathrm{~cm} \times 20.7 \mathrm{~cm}$ (Fig. 2), as well as features suggestive of spontaneous tumour rupture. Splenomegaly, with focal non-enhancing lesions, was also noted on the CT scan.

Further blood tests were done and the most notable findings were as follows: alpha-fetoprotein $10148 \mu \mathrm{g} / \mathrm{L}$; beta-human chorionic gonadotrophin $236 \mathrm{IU} / \mathrm{L}$; alkaline phosphatase $369 \mathrm{U} / \mathrm{L}$; gamma-glutamyl transferase $514 \mathrm{U} / \mathrm{L}$; and aspartate transaminase 283 U/L. Blood tests for malaria and hepatitis A, B and C were all negative. Because of the radiological findings and level of the alpha-fetoprotein the patient was diagnosed with metastatic HCC and assessed to be a candidate for sorafenib, based on the Barcelona Clinic Liver Cancer (BCLC) staging system. She unfortunately declined any further treatment and was lost to follow-up.

\section{Discussion}

The majority of hepatic lesions in pregnancy are benign and often have a good prognosis. However, they pose a diagnostic dilemma as contrast-enhanced imaging in pregnancy is usually avoided owing to concerns of adverse effects on the fetus. There are cases reported in the literature of good maternal and fetal outcomes with early-stage HCC diagnosed in pregnancy, with the mainstay of treatment being surgical resection. ${ }^{[4,5]}$ Metastatic HCC, however, carries a poor prognosis, with an estimated 1-year survival of $<10 \%{ }^{[1]}$ In our case there was delayed presentation and diagnosis, and the patient declined any interventions. The case was also unusual as investigations for viral hepatitis, the most common risk factor for HCC, were negative. Furthermore, it has been recognised that there is an increased incidence of non-AIDS-defining malignancies, including liver malignancies, in HIV-infected persons, although these are often described in the older population. ${ }^{[6]}$ Although anaemia is common in pregnancy and usually has a nutritional aetiology, and thrombocytopenia is not uncommon with advanced HIV disease, their occurrence in pregnancy warrant a full clinical examination and appropriate investigations.

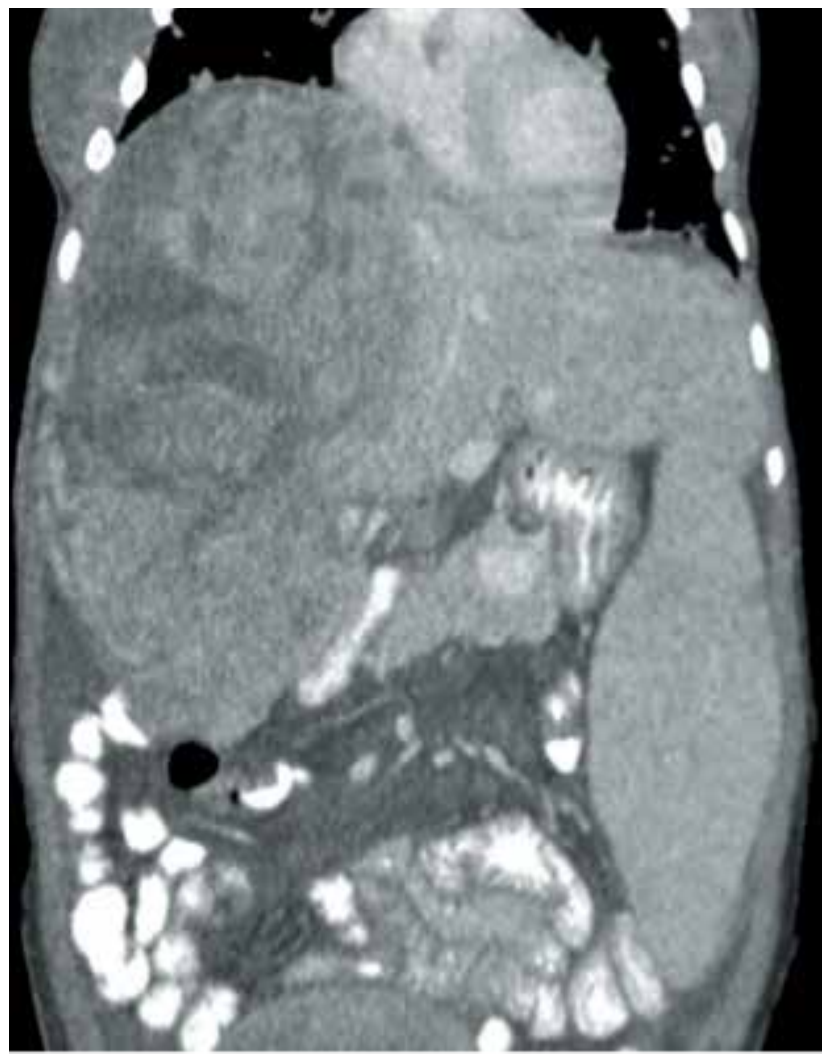

Fig. 2. A CT scan of the abdomen (coronal view), showing a large mass in the right hepatic lobe and splenomegaly. The vascular mass occupies the whole of the right lobe, where dense and hypodense areas are seen within the liver.

\section{References}

1. El-Serag HB. Hepatocellular carcinoma. N Engl J Med 2011;365(12):1118-1127. [http://dx.doi. org/10.1056/NEJMra1001683]

2. Giannitrapani L, Soresi M, La Spada E, Cervello M, D’Alessandro N, Montalto G. Sex hormones 2. Giannitrapani L, Soresi M, La Spada E, Cervello M, D’Alessandro N, Montalto G. Sex hormones
and risk of liver tumor. Ann N Y Acad Sci 2006;1089:228-236. [http://dx.doi.org/10.1196/ annals.1386.044]

3. Choi KK, Hong YJ, Choi SB, et al. Hepatocellular carcinoma during pregnancy: Is hepatocellular carcinoma more aggressive in pregnant patients? J Hepatobiliary Pancreat Sci 2011;18(3):422-431. [http://dx.doi.org/10.1007/s00534-010-0345-6]

4. Chen YY, Chen YR, Wu HK. Hepatocellular carcinoma in pregnancy. J Obstet Gynaecol 2013;33(1):8889. [http://dx.doi.org/10.3109/01443615.2012.721028]

5. Chen HW, Li JY, Huang PQ, Chen RF, Lai EC, Lau WY. Synchronous right hepatectomy and cesarean section in a pregnant lady with hepatocellular carcinoma. Int J Surg Case Rep 2013;4(1):112-114. [http://dx.doi.org/10.1016/j.ijscr.2012.10.014]

6. Wang CC, Silverberg MJ, Abrams DI. Non-AIDS-defining malignancies in the HIV-infected population. Curr Infect Dis Rep 2014;16:406. [http://dx.doi.org/10.1007/s1 1908-014-0406-0] 